\title{
Análisis de la contracción en la fonología prosódica del griego ático
}

\author{
Him Fábrega, Rodrigo \\ Análisis de la contracción en la fonología prosódica del griego ático \\ Synthesis, vol. 26, núm. 2, 2019 \\ Universidad Nacional de La Plata, Argentina \\ Disponible en: http://www.redalyc.org/articulo.oa?id=84662943007 \\ DOI: https://doi.org/10.24215/1851779Xe064
}

Esta obra está bajo una Licencia Creative Commons Atribución-NoComercial-Compartirlgual 4.0 Internacional. 
Artículos

\title{
Análisis de la contracción en la fonología prosódica del griego ático
}

\author{
An Analysis of Contraction in the Attic Greek Prosodic Phonology \\ Rodrigo Him Fábrega \\ Universidad de Panamá, Panamá \\ DOI: https://doi.org/10.24215/1851779Xe064 \\ Redalyc: http://www.redalyc.org/articulo.oa?id=84662943007
}

risraelhim@gmail.com

(iD http://orcid.org/0000-0003-0995-1956

Recepción: 10 Junio 2019

Aprobación: 11 Noviembre 2019

\section{Resumen:}

Este estudio trata sobre la contracción del griego ático, como fenómeno prosódico del estrato léxico. Se impone como meta primaria la de ofrecer una caracterización que pueda servir como trasfondo para la comparación con la crasis, concebida como proceso del estrato post-léxico. La contracción se analiza con los principios de la fonología métrica en función de la descripción de los valores contrastivos del sistema de vocales de la lengua. En la aproximación elaborada, la coalescencia léxica de vocales obedece a la tendencia a eliminar los núcleos de sílaba contiguos, una condición antihiática relacionada con diversos mecanismos de reajuste cuantitativo, que buscan acomodar las sílabas a la longitud máxima de dos moras. Se arguye que el ligamen de los segmentos se realiza de derecha a izquierda, mientras que la propagación de rasgos melódicos se hace en la dirección opuesta, a consecuencia de la primacía del primer núcleo en el entorno del hiato.

Palabras Clave: Griego ático, Contracción, Hiato, Prosodia, Rasgos contrastivos.

\section{ABstract:}

This study deals with Attic Greek contraction, a lexical stratum prosodic phenomenon. Its primary aim is a characterization that supplies the background for a comparison with crasis, conceived as a postlexical process. Contraction is analyzed within the metrical phonology theoretical framework in terms of a previous description of Attic vowel system contrastive features. With this approach, lexical vowel coalescence obeys a tendency to eliminate contiguous syllable nuclei, an antihiatic condition connected with several mechanisms of quantitative adjustment, which adapts the syllables to the maximal permissible length of two moras per nucleus. It is argued that contraction operates from right to left, while melodic feature spreading acts from the opposite direction, as a consequence of the first nucleus primacy in the hiatus situation.

KEYWORDS: Attic Greek, Contraction, Hiatus, Prosody, Contrastive features.

\section{INTRODUCCIÓN}

La contracción y la crasis son fenómenos de coalescencia de vocales que se encuentran en adyacencia inmediata en alguna expresión. En griego antiguo, se admite que obedecen, como en muchas lenguas, a una propensión a suprimir el hiato. ${ }^{1}$ La circunscripción fonológica de los procesos dispone que la contracción se actualice en el estrato léxico: ocurre en el interior de la palabra. La crasis, en cambio, se realiza en el estrato post-léxico: ocurre en la juntura de palabras en la cadena del discurso. ${ }^{2}$

Este estudio se propone elaborar procedimientos para el análisis de la contracción, con la idea de poder emplear dicho recurso en la tarea ulterior de esclarecer el asunto de las convergencias o divergencias con respecto a la crasis en el griego ático del periodo clásico (segunda mitad del siglo $\mathrm{V}$ a. C.). Examina para ello las condiciones en que se produce el fenómeno y las relaciones que guarda con otros atributos fonológicos. En especial, considera las cuestiones concernientes a la dirección en que opera, la clase de los constituyentes prosódicos que selecciona como input, y los rasgos de las estructuras métricas que origina.

La exposición se organiza así: el primer apartado es introductorio. El apartado 2 ofrece las definiciones fundamentales. La sección 3 se interna en el análisis de la contracción, desde una perspectiva que focaliza las 
propiedades prosódicas; de este modo, trata de hacer explícitas las condiciones que la rigen y la clase de las secuencias de segmentos comprendidas en su dominio de aplicación, considerando aspectos tanto cualitativos (vale decir, melódicos), como cuantitativos (vale decir, métricos). El apartado 4 concluye la exposición.

\section{DEFINICIONES FUNDAMENTALES}

La contracción consiste en la coalescencia de vocales concurrentes dentro de una palabra. Supone dos fases: una primera fase en que las vocales se encuentran en hiato, y otra final en que el hiato se suprime mediante la aleación en un producto que en griego ático es regularmente una vocal larga. Se trata, en consecuencia, de un fenómeno del estrato léxico que admite tanto la mezcla de vocales tautomorfémicas, caso de los ejemplos de (1), como la de vocales heteromorfémicas, ${ }^{3}$ caso de los ejemplos de (2).
(1) a. å $\varepsilon \lambda \lambda \circ \mathrm{Q} \rightarrow$ å $\theta \lambda \mathrm{ov}$ ("premio", nom. s.)

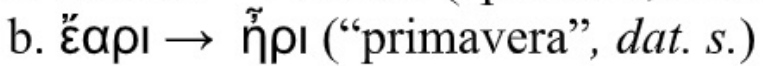
(2) a. $\dot{\varepsilon} \alpha \dot{\omega}(\dot{\varepsilon} \alpha ́+\omega) \rightarrow \dot{\varepsilon} \tilde{\omega}$ ("permitir", ind. act. pte. 1 s.)
b. vóo (vó+o $) \rightarrow$ voũ ("mente", nom. s.)

En (2), el símbolo + denota el linde que separa los morfemas. La flecha horizontal es el símbolo de conversión. En (1) y (2), la flecha señala el paso de la primera a la última fase del proceso de coalescencia.

La crasis, de otra parte, se entiende como coalescencia de vocales en la juntura de dos palabras en el discurso conexo. Fenómeno de la fonología del enunciado, se implementa en el estrato post-léxico, donde por necesidad causa el ligamen de segmentos heteromorfémicos. La coalescencia, también aquí, comprende una primera fase de secuencias en hiato, y produce en la fase final una vocal larga, que sirve de trabazón para las palabras separadas en la primera. El producto es una especie de palabra compuesta creada por sandhi, como se ve en los ejemplos de (3).

\section{(3) a. kaí áyaӨoí $\rightarrow$ káyaӨoí ('y los buenos')

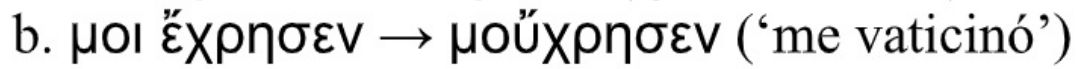 c. ò દ̇uós $\rightarrow$ oưuós ('el mío’)}

De los ejemplos, se desprende que la unión de las vocales de la fase inicial entraña en la crasis la unión de las palabras en la fase final. Este primer atributo se halla asociado con el carácter post-léxico del fenómeno. Además, en la juntura de las palabras en el discurso en griego ático, como en muchas lenguas, la clase de secuencias de vocales difiere de la que agrupa las combinaciones posibles dentro de la palabra simple. Así, aunque contracción y crasis son por igual alteraciones provocadas por el entorno de hiato, la asignación de los procesos a estratos distintos, supone condiciones diferentes de funcionamiento de un tipo de coalescencia con respecto al otro.

\subsection{Definición fónica de las vocales del ático clásico}

La especificación de las propiedades melódicas de las vocales del ático a finales del siglo $\mathrm{V}$ presenta en los análisis disponibles puntos discordantes. ${ }^{4}$ La descripción que aquí se admite es la de Probert (2010, p. 97). La única diferencia radica en la especificación de $\alpha$ con el valor negativo de posterioridad, por considerarla central más que posterior. El conjunto de rasgos fonológicos que emplea la descripción es el de Halle (1995, 
p. 2). El detalle se ofrece en el Cuadro 1. Los enunciados sobre las propiedades fónicas de las vocales del ático que aparecen después ${ }^{5}$ se refieren a las especificaciones de este cuadro.

\section{CUADRO 1}

Valores fónicos de las vocales del ático

\begin{tabular}{|l|l|l|l|l|l|l|l|l|l|l|l|l|l|}
\hline \multirow{2}{*}{ Rasgos melódicos } & \multicolumn{4}{|l|}{ Vocales breves } & \multicolumn{6}{|c|}{ Vocales largas } \\
\cline { 2 - 17 } & L & 0 & $\epsilon$ & 0 & $\alpha$ & $L$ & $u$ & $\epsilon L$ & ou & $\eta$ & $\omega$ & $\alpha$ \\
\hline alto & + & + & - & - & - & + & + & - & - & - & - & - \\
\hline bajo & - & - & - & - & + & - & - & - & - & - & - & + \\
\hline posterior & - & - & - & + & - & - & - & - & + & - & + & - \\
\hline redondo & - & + & - & + & - & - & + & - & + & - & + & - \\
\hline RLA & + & + & + & + & - & + & + & + & + & - & - & - \\
\hline
\end{tabular}

\subsection{Dos modalidades de contracción}

La contracción comprende dos variantes. ${ }^{6}$ La primera, llamada fonética en los estudios de la tradición (y que aquí se denominará fonotáctica), está gobernada por reglas que utilizan esencialmente la clase de información fónica a la que pertenecen los atributos del Cuadro 1. Consiguientemente, las conversiones de los ejemplos de (1) y (2) son fonotácticas en tal sentido. Pero existe también la contracción gramatical, que implica información morfológica más específica que la mera indicación de los lindes del caso heteromorfémico de (2). Tal contracción es siempre heteromorfémica, y no sigue las reglas de la contracción fonotáctica. En (4) aparece un ejemplo de esta modalidad.

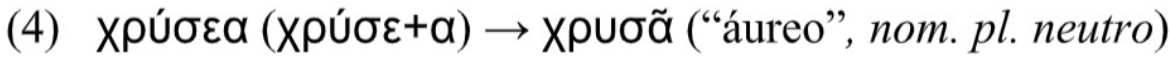

Puede notarse que la contracción de (4) difiere de la que surge en (1b), aunque las vocales que se mezclan son iguales $(\varepsilon, \alpha)$. La contracción de (4) se debe a una analogía con el paradigma de los adjetivos no contractos, en los que el nominativo neutro plural termina por regla en $-\alpha$, como en el caso de $\dot{\alpha} \gamma \alpha \theta \dot{\alpha}$ ("bueno"). ${ }^{7}$ Estas formas de contracción gramatical no serán consideradas en el presente estudio. El análisis examina solo la contracción fonotáctica, que se ejemplifica en las voces de (1) y (2).

\section{Contracción}

En ático, la contracción obedece la serie de condiciones ${ }^{8}$ que se formulan en (5). Recuérdese que los enunciados descriptivos emplean los valores del Cuadro 1.

(5) i. El producto de la coalescencia es una vocal larga.

ii. El producto es redondeado si algún elemento del input es redondeado.

iii. El producto es RLA si algún elemento del input es RLA.

iv. El producto es redondeado si es posterior.

v. El producto es posterior si la vocal inicial del input es posterior. 
La cabal interpretación de esas condiciones supone que la contracción pueda caracterizarse en un nivel más abstracto en la forma que se representa en (6).

\section{(6) i. Fase inicial: $\lambda=\# \ldots V_{1} V_{2} \ldots \#$ ii. Fase final: $\lambda=\# \ldots V_{3} \ldots \#$}

Aquí, el símbolo $\lambda$ denota la unidad de la palabra (por alusión a $\lambda$ ć$_{\circ} \varsigma$ ), V vale por vocal, $V \#$ es una vocal larga (equivalente a dos tiempos o moras, por contraste con la breve, que dura una sola mora). Los subíndices expresan que los segmentos pueden poseer rasgos melódicos diferentes (esto es, $\mathrm{V} \# 3$ puede ser distinta de V1 y de V2 tanto cuantitativa, como cualitativamente). La cantidad de V\#3 refleja una tendencia universal de los procesos de coalescencia. ${ }^{9}$ De acuerdo con (6), la conversión de las vocales en un diptongo (como en el monosílabo oís 'oveja', procedente del bisílabo ơiis) no es una contracción, sino una sinéresis, porque no engendra vocal pura, sino una secuencia de vocal con semivocal. ${ }^{10}$

Las reglas de (5) pueden ser complementadas con las que se enuncian abajo en (7). Algunas de estas han recibido consideración apenas tangencial en la literatura. ${ }^{11}$

(7) i. La primera vocal del input es siempre una vocal pura.

ii. La primera vocal del input, si es larga, nunca contiene los rasgos [+RLA, alto]

iii. El output es alto si la primera vocal del input es alta.

La observación de (7i) no se menciona en ninguno de los estudios sobre el fenómeno de la contracción a que se ha tenido acceso para preparar la presente investigación. Conviene precisar que aquí la expresión vocal pura denota cualquier entidad compuesta de rasgos fonológicos geométricamente estructurados que comprenda al menos el complejo $\{[$-consonántico], Dorsal $\}$, y adopte alguno de los esquemas de (8).

(8)
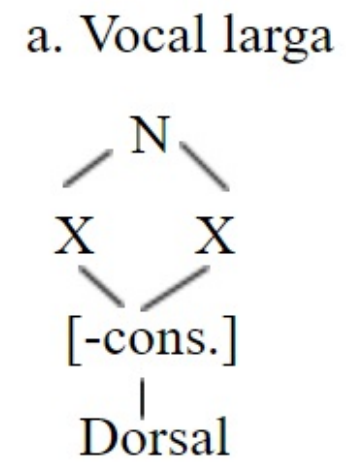

b. Vocal breve

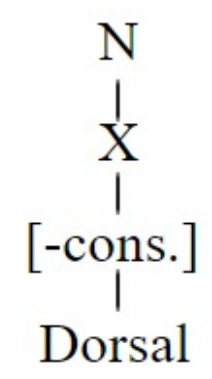

La interpretación de los esquemas es la estándar en fonología métrica: ${ }^{12} \mathrm{~N}$ es un núcleo de sílaba, $\mathrm{X}$ una unidad de medida (equivalente a una mora en el plano de la cadena de tiempos, llamada esqueleto en la literatura), y el conjunto de rasgos fonológicos en la parte inferior constituye un segmento (más específicamente, una vocal). Dado (8), en el input de la contracción, de conformidad con ( $7 \mathrm{i}$ ), no puede haber semivocales en la posición de V1 en (6i). Por tanto, tampoco puede haber diptongos. 


\subsection{Sílaba producto de la contracción}

La descripción que se acaba de proporcionar, presupone que la contracción no se aplica a una cadena lineal de segmentos desprovistos de organización métrica. Por el contrario, el proceso se realiza sobre el armazón de conexiones prosódicas de la palabra. Propiamente, la coalescencia se ciñe a la condición que se formula en (9). Enlazados con sendos núcleos de sílaba $(\mathrm{N})$ y sendas ranuras de la secuencia de tiempo (X), en (9) V1, V2 se usan como signos taquigráficos del conjunto de rasgos melódicos que conforman una vocal pura. $\mathrm{R}$ representa la rima o extremo final de la sílaba, la cual domina al núcleo. La $\sigma$ es el símbolo de la sílaba, que puede ser expandida con un inicio (I) y una coda (C), los cuales no se incluyen en (9). El asterisco sobre el segundo $\mathrm{N}$ denota la impropiedad de la configuración que comprende dos núcleos contiguos.

\section{(9) Condición Antihiática}

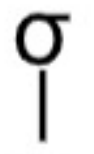

$\mathrm{R}$
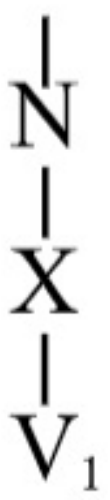

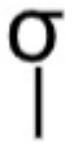

$\mathrm{R}$
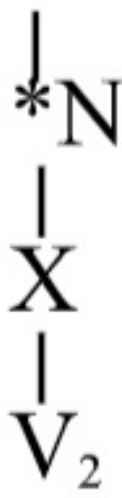

La Condición Antihiática expresa la tendencia, observada en muchas lenguas, a excluir cimas de sílaba adyacentes, por tratarse de una secuencia que no satisface las propiedades de la estructura prosódica óptima, en la que la hay alternancia de sonoridad entre las unidades implementada por la alternancia de vocales y consonantes según el esquema (C)V.CV, con el punto como marca del límite de sílaba. ${ }^{13}$

La coalescencia de vocales es una consecuencia de la condición de (9), que en el caso del griego ático busca acomodar los elementos según un patrón fonotáctico cónsono con la alternancia rítmica que reclama puntos de menor prominencia entre los momentos en que ocurren las cumbres de sonoridad. Un primer paso hacia dicho objetivo consiste en la supresión del segundo núcleo y la reasociación subsecuente de los atributos que se hallan en su dominio. Es lo que reflejan los diagramas de (10). 
(10)
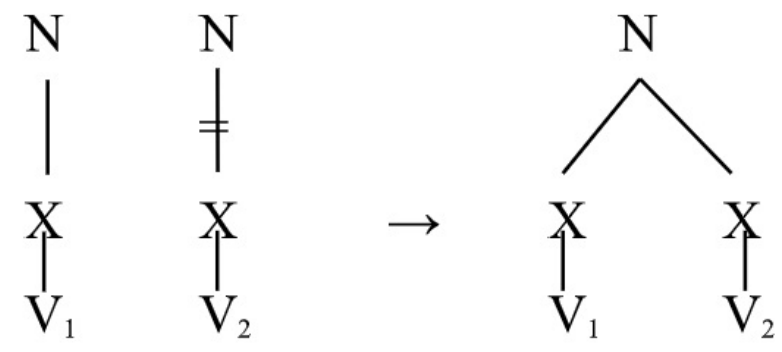

El signo = que cruza la línea de asociación indica el desenganche de los nodos. Ahora bien, un núcleo que no contenga semivocales demanda un único conjunto de rasgos melódicos, por lo cual el producto de (10) se reagrupa, de conformidad con (5i) (6) y (8), como se indica en (11). Donde, V\#3 viene a ser el producto de la contracción de V1 y V2. Así, un problema central del proceso consiste en establecer cómo se especifica V\#3.

(11)
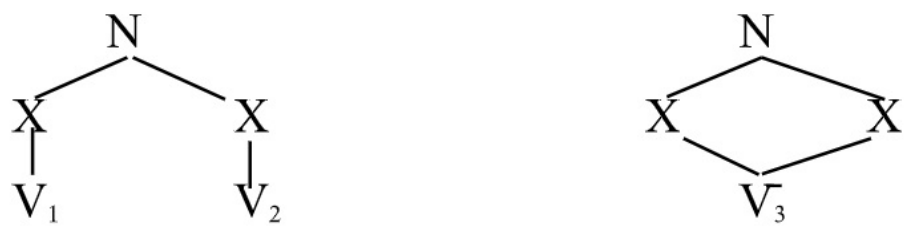

\subsubsection{Ilustración}

Considérese la palabra de (1b) ह̌apl ("primavera”, dat. s.). Esta responde a la descripción de (12), desde la cual, por lo que se sigue de (9) y (10), se obtiene (13).

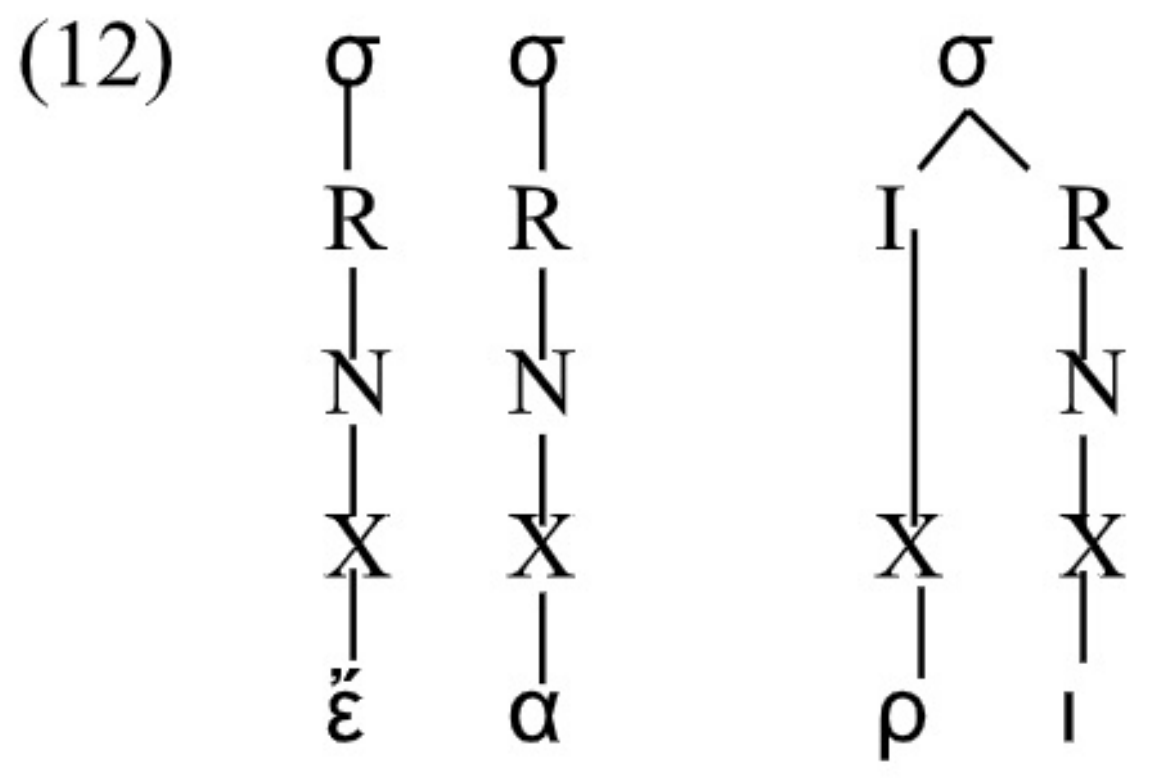



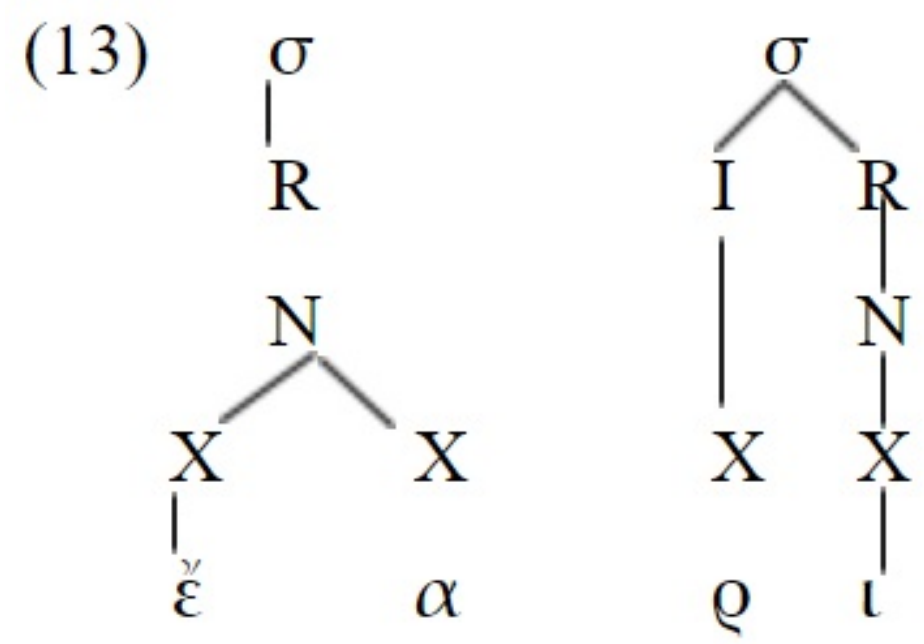

Luego, según (13), se deriva (14).
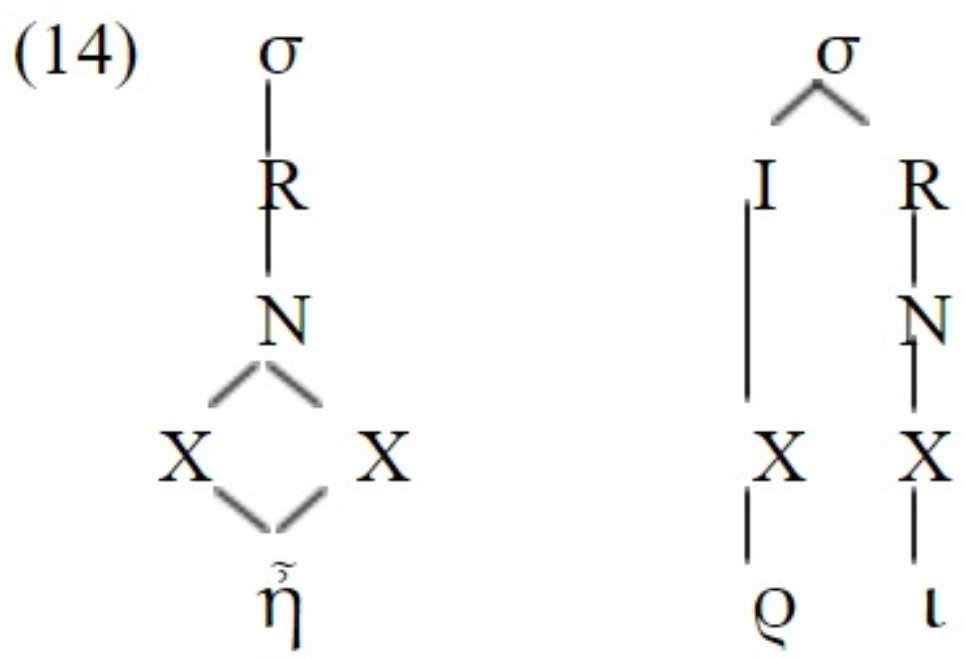

El contenido melódico del producto, el paso de $\tilde{\varepsilon}-\alpha$ hacia $\tilde{\eta}$, constituye el problema de especificación que se mencionó en relación con (11): la especificación de V\#3. El acento suele permanecer en la coalescencia sobre la mora que lo porta en la forma no contracta.

Un aspecto importante del cambio ocasionado por la Condición Antihiática es que el desgajamiento de la ranura temporal se realiza en el núcleo final de la secuencia transgresora. Tal característica puede ponerse en relación con el hecho de que el hiato surge debido a la estructura defectiva de la sílaba que contiene dicho núcleo: la misma carece del margen consonántico del prototipo universal $\mathrm{CV},{ }^{14}$ y entraña la disrupción de la alternancia rítmica del grado de sonoridad. Es cierto que la sílaba del comienzo de éapı tampoco satisface el prototipo, pero es la defectividad de la segunda la que provoca la pérdida de la alternancia que proscribe la Condición Antihiática.

\subsection{Direccionalidad}

La dirección en que se efectúa el reenganche de la unidad desgajada en (10) puede entenderse como signo del modo en que funciona la contracción. La coalescencia procede en general de derecha a izquierda, comenzando 
desde el extremo final de la palabra. Este modo de direccionalidad se confirma al examinar las coalescencias de las formas incluidas en (2). En el caso de $\dot{\varepsilon} \dot{\alpha} \omega$ ("permito"), verbigracia, si se comienza desde la izquierda se obtiene la derivación impropia de (1), mediante la unión de $\dot{\varepsilon} \operatorname{con} \dot{\alpha}$.

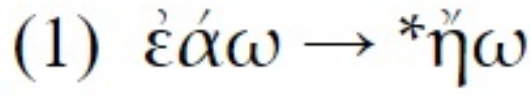

Por otro lado, la forma contracta voṽ ("mente”, gen. s.) implica, al menos diacrónicamente, una coalescencia doble en la dirección de derecha a izquierda, como se ilustra paso por paso en (16).

$$
\begin{aligned}
& \text { (16) i. input: vó }+ \text { o }+ \text { o (raíz }+ \text { vocal de unión }+ \text { gen. }) \\
& \text { ii. Primera contracción: vó }+ \text { ov } \\
& \text { iii. Segunda contracción: vov }
\end{aligned}
$$

El cambio de (16i) a (16ii) refleja un proceso que distingue la fase del griego homérico (y micénico) de la del jónico-ático clásico. El paso de (16ii) a (16iii) constituye la asociación sincrónica del período clásico entre la forma no contracta (aún corriente en la variante jónica) y la forma contracta característica del ático. ${ }^{15}$

Supóngase, por contraste, que a partir de (16i) se efectúa la coalescencia de izquierda a derecha. Ello produciría la forma * voṽo, desde la cual no habría paso hacia la contracta voṽ, porque en griego ático no hay contracciones que tengan el segmento ov $(=[\mathrm{o}:])$ como primera vocal del input, de acuerdo con lo que enuncia el condicionamiento de (7ii).

En resumen, la contracción en ático observa dos modos de direccionalidad: (i) el reenganche de elementos destrabados se realiza de derecha a izquierda; (ii) la coalescencia procede de derecha a izquierda, desde el final de la palabra.

\subsection{Rasgos melódicos contrastivos}

En el Cuadro 1, las vocales griegas se describen en un nivel fonético: la descripción comprende atributos tanto contrastivos como atributos que carecen de pertinencia para diferenciar los segmentos. De este conjunto, solo es necesaria la información contrastiva para caracterizar el producto de la coalescencia. La especificación de rasgos contrastivos se ofrece en el Cuadro 2. 
CUADRO 2

Valores fónicos contrastivos de las vocales del ático

\begin{tabular}{|c|c|c|c|c|c|c|c|c|c|c|c|c|}
\hline \multirow{2}{*}{$\begin{array}{l}\text { Rasgos } \\
\text { melódicos }\end{array}$} & \multicolumn{5}{|c|}{ Vocales breves } & \multicolumn{7}{|c|}{ Vocales largas } \\
\hline & L & $v$ & $\epsilon$ & 0 & $\alpha$ & L & v & $\epsilon \mathrm{L}$ & OU & $\eta$ & $\omega$ & $\alpha$ \\
\hline alto & + & + & - & - & $\langle-\rangle$ & + & + & - & - & $\begin{array}{l}<- \\
>\end{array}$ & $\longleftrightarrow-〉$ & $\langle->$ \\
\hline bajo & $\langle-\rangle$ & $\langle->$ & $\longleftrightarrow->$ & $\langle-\rangle$ & + & $\langle-\rangle$ & $\langle-\rangle$ & $\langle-\rangle$ & $\langle-\rangle$ & - & - & + \\
\hline posterior & - & - & - & + & $\langle\rightarrow\rangle$ & - & - & - & + & - & + & $\langle-\rangle$ \\
\hline redondo & - & + & $\longleftrightarrow->$ & $\langle+\rangle$ & $\langle-\rangle$ & - & + & $\longleftrightarrow->$ & $+>$ & $\begin{array}{l}<- \\
>\end{array}$ & $\langle+\rangle$ & $\langle->$ \\
\hline RLA & $\langle+\rangle$ & $\langle+\rangle$ & + & + & - & $\langle+\rangle$ & $\langle+\rangle$ & + & + & - & - & - \\
\hline
\end{tabular}

Aquí se adopta la convención de encerrar entre paréntesis angulares los valores no pertinentes. Se obtiene así una descripción que permite poner en contraste cada vocal con el resto de las vocales del sistema. Recuérdese que $\alpha$ se especifica como [-post.], en razón de que se otorga prevalencia a la posición central en que se articula. Dicha centralidad es un presupuesto de los diagramas triangulares empleados para representar el espacio articulatorio de las vocales áticas. ${ }^{16}$

Para proceder con el análisis de la contracción con el apoyo de los datos del Cuadro 2, será conveniente dividir las secuencias de acuerdo con sus propiedades melódicas y prosódicas. Resultan así las subclases siguientes: (i) secuencias de vocales iguales, (ii) de vocales breves diferentes, (iii) de vocales diferentes, una de las cuales es larga, (iv) secuencias que incluyen diptongos. Se tratan a continuación, en el orden dado.

\subsection{Secuencias de vocales iguales}

La igualdad en este caso se establece en función de la cualidad, y la cantidad no modifica el producto, salvo en lo que atañe a la condición de (5i), que demanda vocal larga en el output. Dicha condición en general es cónsona con el Principio de Contorno Obligatorio, según el cual las vocales largas tienen la estructura prosódica de un segmento (un elemento melódico) asociado con dos posiciones del esqueleto (dos tiempos o moras). ${ }^{17}$ Los ejemplos de (17) proporcionan la confirmación. Las expresiones entre barras a la derecha dan la transcripción fonológica estándar (Alfabeto Fonético Internacional) de cada expresión. Se incluyen para mostrar más claramente los valores de las unidades. Concretamente, el vocablo de (17a), en la secuencia que sufre contracción, se deriva según lo expuesto como se indica abajo (se omiten detalles). La tilde tras la primera unidad de medida en la fila del esqueleto $\left(\mathrm{X}^{\prime}\right)$ indica dónde se ubica el acento. 


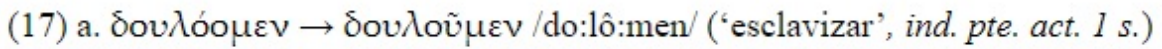

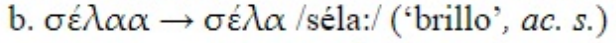

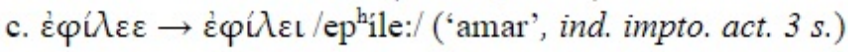

i. Input:

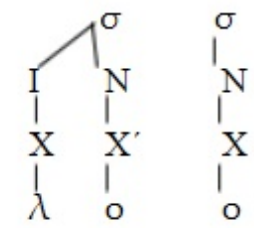

ii. Coalescencia (por la Condición Antihiática):

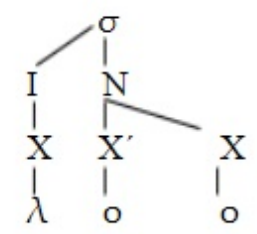

iii. Producto (por el Principio de Contorno Obligatorio):

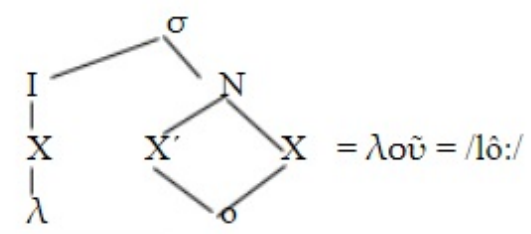

Similarmente, para los otros casos de vocales largas derivadas.

\subsection{Secuencias de vocales breves distintas: Reglas de propagación}

Los datos relevantes se despliegan en (19):

(19) a. $\tau^{\prime} \mu \alpha \varepsilon \rightarrow \tau i ́ \mu \alpha /$ tíma:/ ("honrar", imp. act. 2 s.)

b. $\tau \mu \alpha \dot{\alpha} о \mu \varepsilon \nu \rightarrow \tau \iota \mu \tilde{\omega} \mu \varepsilon \nu / t i m{ }^{\wedge}: \mathrm{men} /$ ("honrar", ind. pte. act. $1 \mathrm{pl}$.)

c. $\alpha \mathrm{i} \delta o ́ \alpha \rightarrow \alpha \mathrm{i} \delta \tilde{\omega} /$ aido":/ ("vergüenza", ac. s.)

d. $\delta$ oú $\lambda o \varepsilon \rightarrow \delta o u ́ \lambda o v /$ dó:lo:/ ("esclavizar", imp. act. 2 s.)

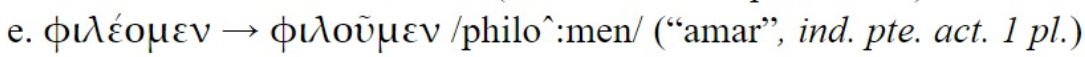

La primera observación es que las vocales no se contraen en igual escala o con la misma consistencia. Las vocales altas, en especial, muestran una conducta desviante, y si llegan a contraerse, el fenómeno ocurre muy esporádicamente, con apego a la regla de (7iii). Tal comportamiento reclama investigación ulterior. ${ }^{18}$

Las coalescencias de (19) proporcionan el caso general del grupo en que se contraen vocales breves diferentes. Los ejemplos revelan, de conformidad con los enunciados de (5), la prevalencia de los valores contrastivos [+ posterior] y [-RLA]. El producto puede derivarse propiamente con las reglas de propagación que se formulan en (20). Para las mismas, vale la condición implícita, por lo dicho arriba, de que en los segmentos pertinentes no se incluye la especificación [+alto]. 
(20) Reglas de propagación de la coalescencia léxica:

En una estructura de dos conjuntos de rasgos melódicos contiguos de tipo $\{[$-cons.], Dorsal $\}$ asociados con sendas ranuras del esqueleto dominadas por el mismo núcleo, como en el output de (10):

i. Propáguese [+post.] de izquierda a derecha, o en su defecto, de derecha a izquierda.

ii. Propáguese [-RLA] de izquierda a derecha, o en su defecto, de derecha a izquierda.

iii. Propáguese [+bajo] de izquierda a derecha si los segmentos comparten los atributos [-RLA, -post.].

Las reglas de (20i) y (20ii) recubren dos operaciones de dispersión, una en dirección hacia la derecha, y otra en sentido inverso. La regla de (20iii) se aplica al producto de (20ii), que la precede en orden de aplicación. En (21) se ofrece la derivación de las contracciones de (19d) y (19e), como ilustración. La flecha oblicua indica la dirección de la propagación. Se omiten detalles. ${ }^{19}$

\section{(21) i. Input:}
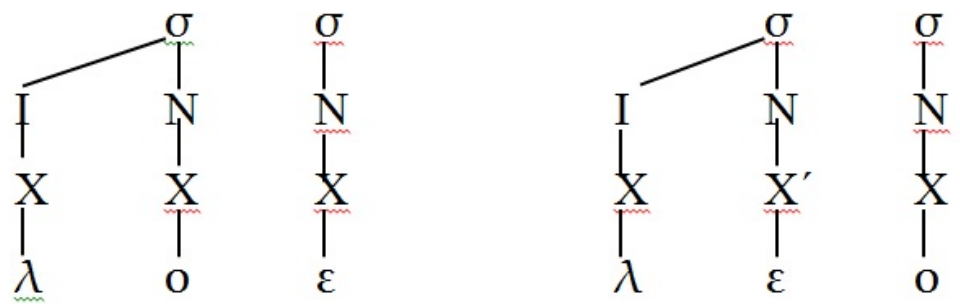

ii. Propagación(20i):
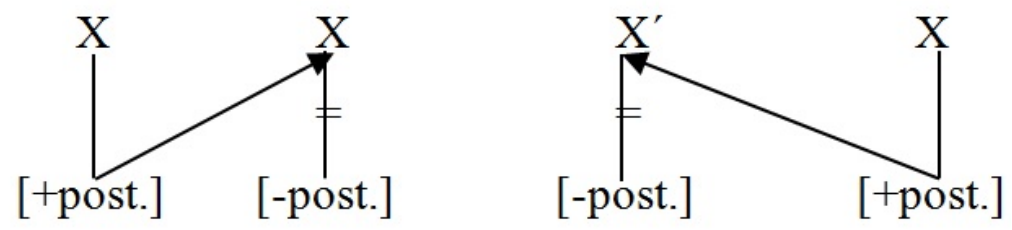

iii. Output, aplicando Principio de Contorno Obligatorio:
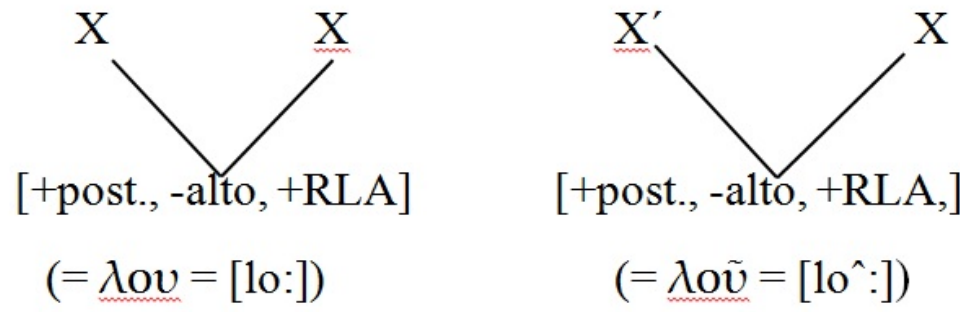

La expresión de (19b) se analiza de manera análoga. Para obtener (19a), en cambio, se necesita la regla de (20iii). Así, por (20ii), se llega a la configuración de (22), desde el original $\tau i$ i $\alpha$. 
(22)

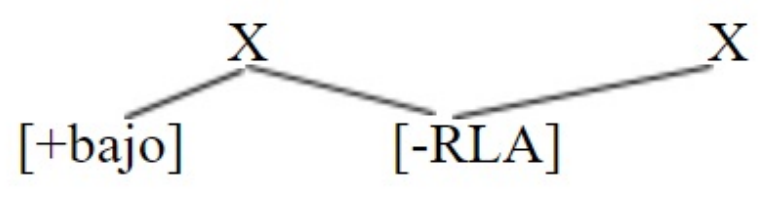

Desde la cual, por (20iii), sale (23), en la contracta $\tau i \mu \alpha$ ('respeta', imp.)
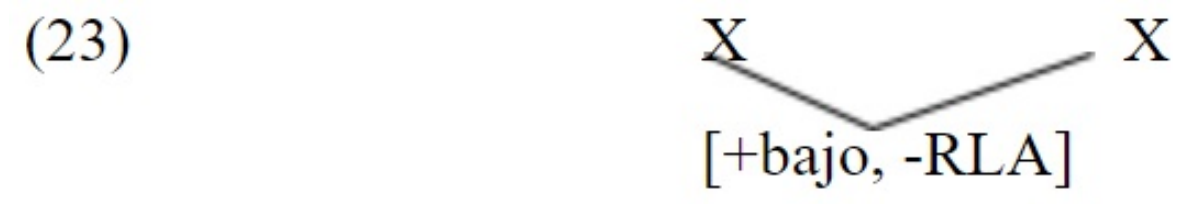

$$
(=\bar{\alpha}=[\mathrm{a}:])
$$

Considérese ahora la contracción de (19c). Para producirla es preciso añadir una regla a la serie de (20). El recurso parece el inverso de (20iii), porque se aplica hacia la derecha, y en vez de propagar, destraba el rasgo [+bajo]. Se caracteriza en (24).

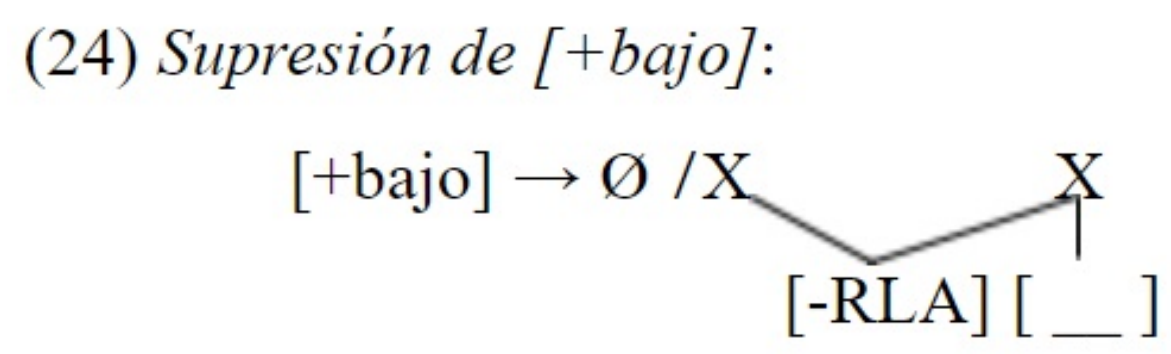

Crucialmente, (24) no solo da cuenta de casos como el de (19c): a la vez, permite tratar términos como $\tau \varepsilon i \chi \varepsilon \alpha$ ("muro", ac. pl. neutro), forma contracta $\tau \varepsilon i \chi \chi \eta$, que no se acomodan al molde de las reglas de (20). Las derivaciones de $\alpha i \delta \tilde{\omega}$ y $\tau \varepsilon i \chi \eta$ se dan abajo. ${ }^{20}$ Interesa anotar que en todas las derivaciones elaboradas hasta aquí solo se emplean los rasgos contrastivos del Cuadro 2. Las coalescencias que no se avienen con las pautas trazadas quedan recluidas en el grupo de las contracciones gramaticales y reclaman tratamiento especial. La cuestión no será abordada en este estudio. 
(25) i. Input:

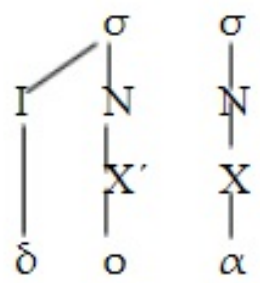

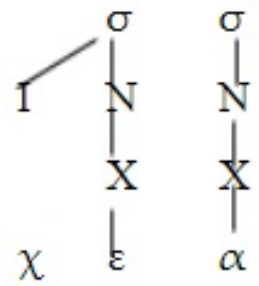

ii. Propagación (20i) y (20ii):
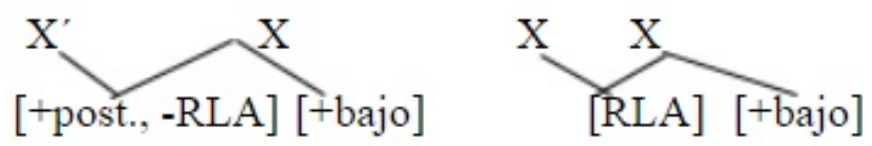

iii. Supresión de [+bajo], (24):
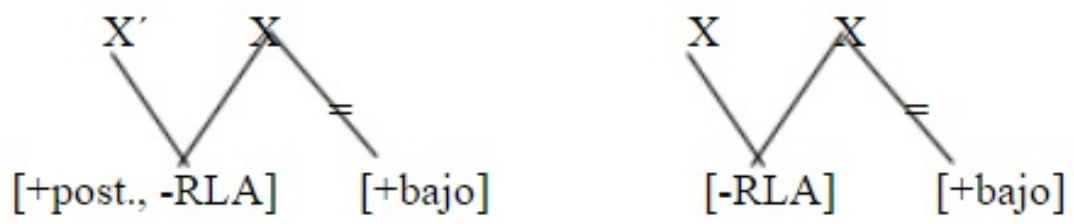

iv. Producto (Principio de Contorno Obligatorio):

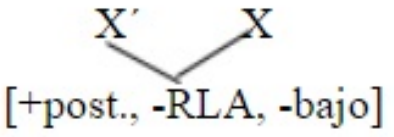

$$
\left(=\delta \tilde{\omega}=\left[\mathrm{do}^{\wedge}:\right]\right)
$$

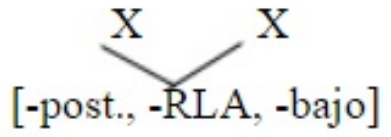

$\left(=\chi \eta=\left[\mathrm{k}^{\mathrm{h}} \varepsilon\right]\right)$

\subsection{Secuencias que comprenden una vocal larga}

Las combinaciones de segmentos en que consta una vocal larga responden a los mismos condicionamientos formulados en la sección anterior, y en especial a las reglas de (20) y (24). Solo debe añadirse un instrumento de ajuste métrico que convierte cualquier núcleo que congregue más de dos medidas de tiempo (dos moras), a la cantidad canónica de las dos unidades. Las expresiones con exceso extremo agrupan cuatro ranuras temporales. Por ejemplo, la no contracta de (26).

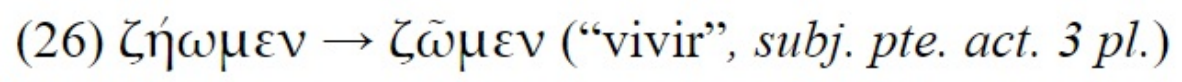

Aquí, tras el cambio ocasionado por la Condición Antihiática, ocurre la reducción de moras que ajusta la cantidad del producto. Así: 
(27)

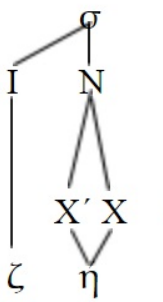

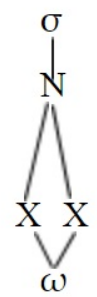
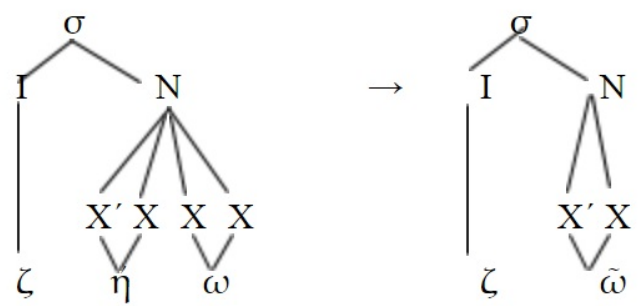

La conversión obedece la constricción de exceso de moras que se enuncia en (28).

\section{(28) Condición de Exceso de Moras}

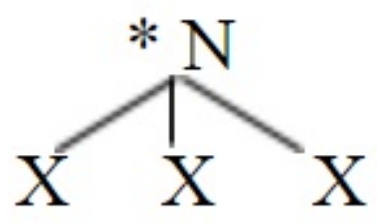

Propiamente, esta constricción se aplica al resultado de las operaciones de propagación. Por tanto, su input en la derivación de (27) es (29).
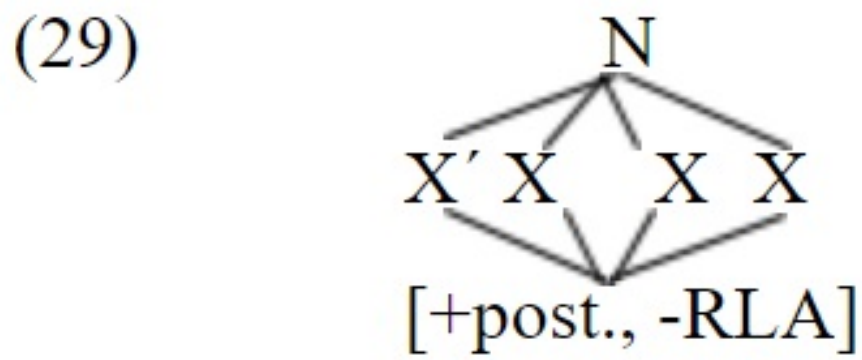

Sobre esta estructura, Exceso de Moras (28) provoca el arreglo que se muestra en (30). Importa notar que las ranuras que se suprimen son las que ocupan la zona interna de la secuencia, y que el proceso ocurre iterativamente.

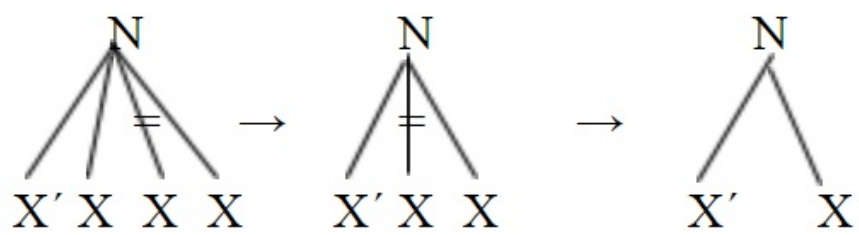

El producto del proceso, representado de manera abreviada en (27), en consonancia con lo que dispone el Principio de Contorno Obligatorio, sería el siguiente: 
$(31)$

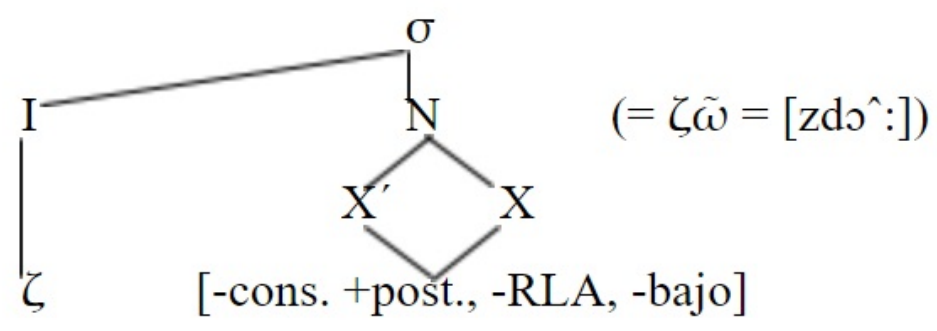

Similarmente para otras coalescencias que admiten vocales largas, como las de (32).

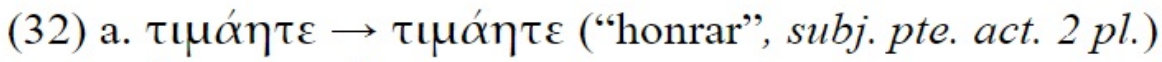

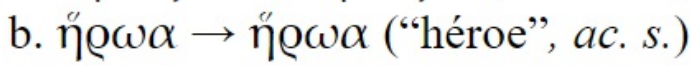

En estas coalescencias, dado que en el input hay solo tres ranuras de tiempo, Exceso de Moras se aplica una sola vez. En el caso de (32a), se requiere la regla de propagación de (20iii), mientras que en (32b) interviene la regla supresora de sentido inverso de (24).

\subsection{Secuencias con diptongos}

En ático, una expresión que incita una coalescencia solo puede incluir diptongo en la posición del segundo núcleo, por las constricciones que se desprenden de (7i) y (8). Tales combinaciones se analizan con los mismos recursos empleados para producir las contracciones de vocales largas. De hecho, proveen la confirmación de que las ranuras métricas que se destraban cuando se excede el límite de moras son las que ocupan la zona interna de la secuencia. Considérese, en conexión con ello, los ejemplos de (33).

$$
\begin{aligned}
& \text { (33) a. vóot } \rightarrow \text { voĩ ('mente', nom. pl.) }
\end{aligned}
$$

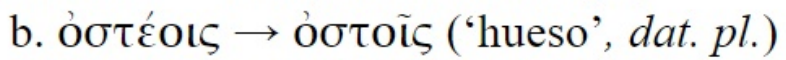

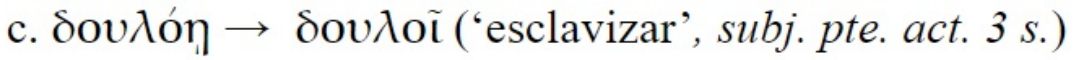

Los casos de (33a) y (33b) son análogos. En (33a) ocurre una combinación de vocales cerradas iguales, de las que se desgaja la interna en el producto. En el caso de ỏ $\sigma \dot{\varepsilon} \circ \iota_{\varsigma}$, las reglas de propagación engendran una doble asociación con los mismos rasgos, y Exceso de Moras recorta la configuración. La derivación simplificada se da en (34). 
(34) i. Input:
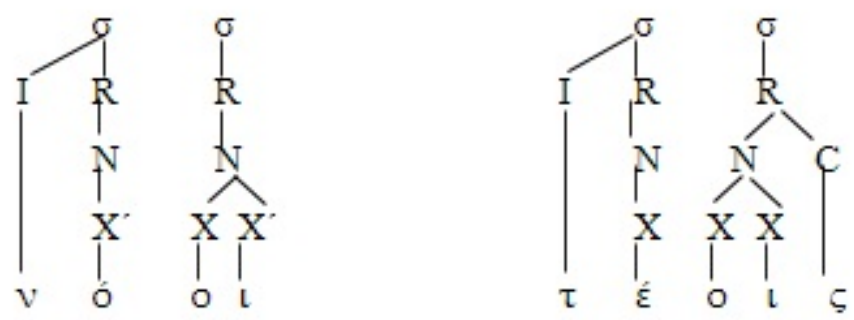

ii. Por Condición Antihiática (9) y Propagación (20):
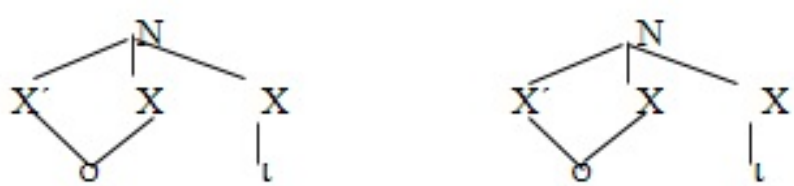

iii: Por Exceso de Moras, (28):
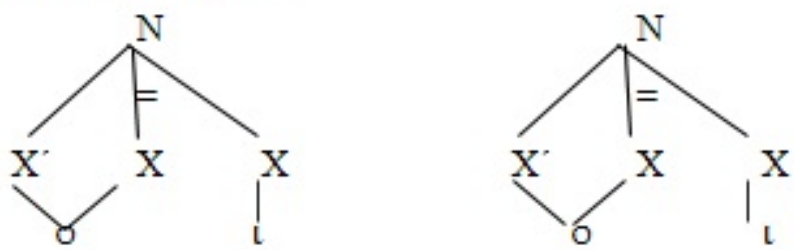

iv. Producto:

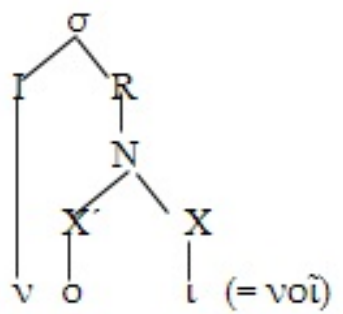

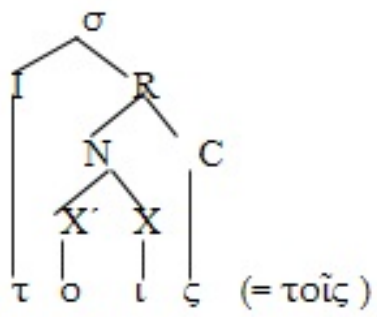

En el producto, la iota es segundo miembro de diptongo. Apareciendo aquí como semivocal, puede combinarse con una vocal pura en un núcleo complejo como el de (34iv), que no se amolda a los esquemas de la contracción, formulados en (6) y (11). En (34iv) se produce una sinéresis.

El ejemplo de (33c) corresponde a la secuencia de cuatro moras, de las que se desgajan las dos internas. El mismo hace patente que la vocal alta del diptongo en la sílaba final se admite sin estorbo, por no hallarse contigua al núcleo de la penúltima sílaba. Además, el proceso revela que en la representación subyacente de la palabra dicha vocal se adjudica una posición métrica (un tiempo en el esqueleto). La derivación confirma la

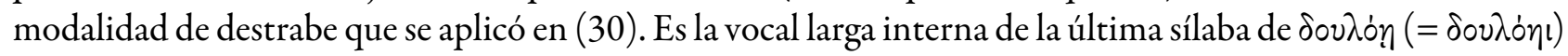
la que se elimina. Así (detalles omitidos): 
(35) i. Input:
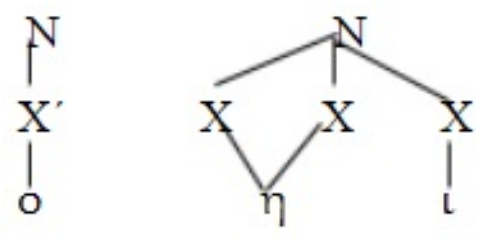

ii. Por Condición Antihiática:

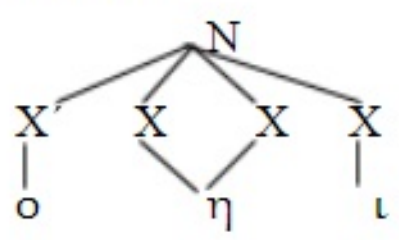

iii. Por Exceso de Moras:

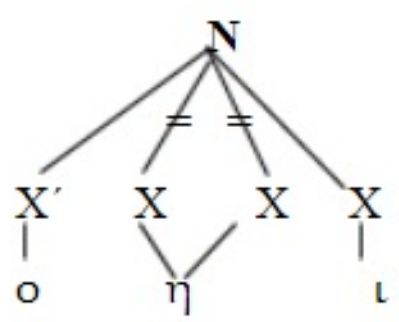

iv. Producto:

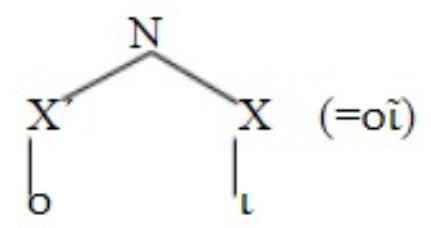

\subsection{Traslado de núcleo a coda}

Ejemplos como el de (33c) traen a la superficie la situación en que los fenómenos de reajuste prosódico se constriñen entre sí. De este modo, en la derivación de (35) no se observan los resultados posee vocales breves abiertas (es decir, vocales breves con valor [-RLA]). Esta restricción excluye el output ${ }^{*}\left[\#_{l}\right]$ que saldría de la propagación. La impropiedad se resuelve eliminando las moras superfluas de la $\eta$ que ha entrado a ser parte del núcleo expandido en (35ii).

Pero la última sílaba de (33c) encubre un recurso alternativo para suprimir la sobreabundancia de moras, que consiste en trasladar una vocal alta a la posición de coda, lo cual equivale a suprimir la ranura de tiempo que le correspondía sin eliminar el segmento. El mismo pierde su condición de vocal pura para adoptar la de las consonantes. Esto ocurre, verbigracia, en $\lambda \hat{o} \gamma \omega$ (de $\lambda \dot{o} \gamma \omega \iota$, a su vez de $\lambda \circ \gamma \circ \varepsilon l$ ), donde la secuencia contracta de tres moras $\omega \iota$ pasa a $\omega$ p por cambio de vocal pura a semiconsonante (traspaso a coda). Más explícitamente: 

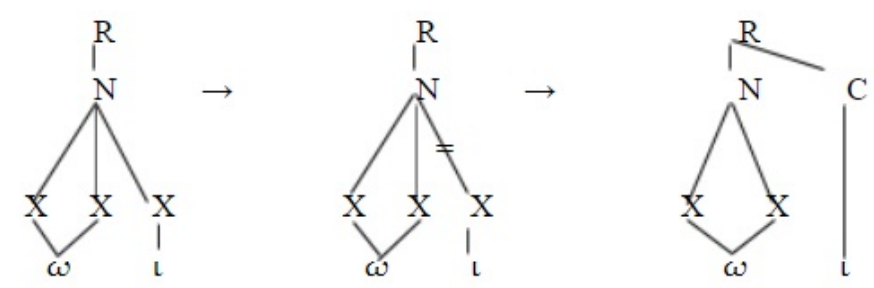

La derivación es característica de la conjugación de los verbos en $-\alpha$, en la circunstancia en que la reducción de los núcleos comporta una serie de tres moras, como se ve en $\tau \mu \dot{\alpha} \varepsilon \iota \rightarrow \tau \psi \mu \tilde{\alpha}$ ("honrar", ind. pte. act. 3 s.), y

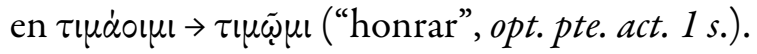

La condición de traspaso a coda se define más específicamente en (37).

\section{(37) Condición de Traspaso de Núcleo Coda: En el entorno de sobreabundancia de no más que una mora, engánchese una vocal alta a la coda siempre que sea posible.}

Existen, en consecuencia, dos instrumentos para reparar el rebasamiento del límite de las dos moras, a saber: (i) El desenganche de las moras internas mediante Exceso de Moras, (28), como ocurre en la derivación de (35) con la supresión de $\eta$; (ii) el traslado de una vocal alta, transformada en semiconsonante, a la posición de la coda, según lo que estipula la condición de (37), como ocurre en la derivación de (36).

La derivación de (35) muestra que los dos procesos de recorte de moras no pueden intervenir en la misma derivación. En (35), Traspaso de Núcleo a Coda, caso de que pudiera aplicarse, no resolvería el problema de sobreabundancia de tiempos, cualquiera que sea el orden en que se disponga con respecto a las reglas de propagación. Sea que se aplique antes o que lo haga después, la derivación engendra una secuencia que aún rebasa el límite de las dos moras. En (35), en tal sentido, se implementa la modalidad de derivación más económica: aquella que aplica solo uno de los recursos de reajuste métrico. La falta de solidaridad entre los procesos se registra formalmente en la cláusula que constriñe el traspaso a la coda al entorno de rebasamiento mínimo de una mora. ${ }^{21}$

\section{Conclusión}

La coalescencia del estrato léxico del griego ático responde a una constricción antihiática que reaparece en muchas lenguas. Si se admite que las vocales del ático poseen los valores contrastivos que se despliegan en el Cuadro 2, entonces el fenómeno de la contracción puede ser descrito satisfactoriamente con el recurso de las operaciones de propagación de los rasgos [+post.], [-RLA] y [+bajo], formuladas en (20), más el complemento de la regla de desenganche de [+bajo] dada en (24). Para las secuencias que incluyen vocales largas o diptongos es preciso añadir dos procesos de reducción de moras. Exceso de Moras suprime las ranuras superfluas en la zona interna de la fila de unidades métricas, mientras que Traspaso de Núcleo a Coda reubica una vocal alta en la posición de la coda. Ambos recursos traen como consecuencia el reajuste de la cantidad de la sílaba al límite máximo que estipula el sistema.

Se ha mostrado también que la contracción procede en ático desde el final de la palabra, en dirección de derecha a izquierda, y en consonancia con las generalizaciones formuladas en (7i) y (7ii), que restringen las combinaciones de vocales en el input. El análisis ofrecido es válido, en general, para el grupo de las contracciones fonotácticas. Las contracciones gramaticales no se ciñen a los condicionamientos expuestos, y reclaman tratamiento especial. Queda por dilucidar si, como se afirma en algunas obras, ${ }^{22}$ la crasis puede ser 
descrita, contra lo que sugiere la pertenencia a un estrato distinto, con las herramientas diseñadas para dar cuenta de la contracción, una cuestión que también pide ulterior estudio.

\section{REFERENCIAS}

Arhens, H. (1845). De crasi et aphaeresi. Stolberg: Stolbergae Hercynicae.

Berenguer-Sánchez, J. (2011). Notas sobre la «ley de alargamiento de Wackernagel». Emérita, 79, 381-390.

Brandenstein, W. (1964). Lingüistica griega. Madrid: Gredos.

Casali, R. (1996). Resolving Hiatus (Tesis doctoral). University of California, Los Angeles.

Casali, R. (2011). Hiatus Resolution. En M. van Oostendorp et al (Ed.), The Blackburn Companion to Phonology (pp. 1434-1460). Chichester: Wiley-Blackwell.

Chantraine, P (1974). Dictionnaire étymologique de la langue grecque, Tome III. Paris: Editions Klincksieck.

Clements, G. (1990). The role of the sonority cycle in core syllabification. En J. Kingston y M. Beckman (Ed.), Papers in Laboratory Phonology I (pp. 283-333). Cambridge: Cambridge University Press.

De Haas, W. (1988). A formal theory of vowel coalescence: A case study of Ancient Greek. Dordrecht: Foris.

Devine, A. y Stephens, L. (1994). The prosody of Greek speech. Oxford: Oxford University Press.

Grammont, M. (1948). Phonétique du grec ancient. Lyon: IAC.

Halle, M. (1995). Feature Geometry and Feature Spreading. Linguistic Inquiry, 26, 1-46.

Halle, M., Harris, J. y Vergnaud, J. R. (1991). A Reexamination of the Stress Erasure Convention and Spanish Stress. Linguistic Inquiry, 22, 141-159.

Harris, J. (1996 2 ). Integrity of Prosodic Constituents and the Domain of Syllabification Rules in Spanish and Catalan. En K. Hale y S. Keyser (Ed.), The View from Building 20: Essays in Linguistics in Honor of Sylvain Bromberger (pp. 177-193). Cambridge, Massachusetts: MIT Press.

Hayes, B. (1989). Compensatory Lengthening in Moraic Phonology. Linguistic Inquiry, 20, 253-306.

Hermann, G. $\left(1852^{3}\right)$. Epitome doctrinae metricae. Lipsiae: Apud G. Fleischerum.

Horrocks, G. $\left(2010^{2}\right)$. Greek: A history of the language and its speakers. Chichester/Malden: Wiley-Blackwell.

Jakobson, R. (1984/1963). Importancia de los universales del lenguaje para la lingüística. En Ensayos de lingüistica general (pp. 47-65). Barcelona: Ariel.

Jakobson, R. y Halle, M. (1967/1956). Fundamentos del lenguaje. Madrid: Ciencia Nueva.

Kiparsky, P. (1985). Some Consequences of Lexical Phonology. Phonology Yearbook, 2, 85-138.

Kühner, R. y Blass, F. (1890). Ausführliche Grammatik der Griechischen Sprache. Hannover: Hahnsche Buchhandlung. Lejeune, M. (1987/1967). Phonétique historique du mycénien et de grec ancient. Paris: Klincksieck.

McCarthy, J. (1986). OCP effects: Gemination and antigemination. Linguistic Inquiry, 17, 207-263.

McCarthy, J. y Prince, A. (1996/1986). Prosodic morphology. University of Massachusetts, Amherst y Rutgers University, New Brunswick.

Mohanan, K. (1986). The theory of Lexical Phonology. Dordrecht: Reidel.

Monro, D. (1891). A Grammar of the Homeric Dialect. Oxford: Clarendon Press.

Palmer, L. R. (1962). The language of Homer. En A. Wace y F. Stubbings (Ed.), A companion to Homer (pp. 75-178). London: Macmillan.

Probert, Ph. (2010). Phonology. En E. Bakker (Ed.), A Companion to the Ancient Greek Language (pp. 85-103). Chichester/Malden: Wiley-Blackwell.

Rubach, J. (1998). A Slovak argument for the onset-rhyme distinction. Inquiry Linguistic, 29, 168-179.

Smyth, H. (1920). A Greek Grammar for Colleges. New York: American Book Co.

Sommerstein, A. (1973). The sound pattern of Ancient Greek. Oxford: Basil Blackwell. 
Waeschke, H. (1875). De crasi aristophanea. Dessaviae: Apud Aemilium Barthium.

Yip, M. (1988). The Obligatory Contour Principle and Phonological rules: A loss of identity. Inquiry Linguistic, 19, 65-100.

\section{Notas}

1 Arhens (1845, pp. 1-3), Hermann (1852, p. 26), Smyth (1920, p. 18), Lejeune (1987, p. 258), De Haas (1988, p. 77), Casali (1996, p. 1), Berenguer-Sánchez (2011, p. 388).

2 Cf. Kiparsky (1985, pp. 86-87), Mohanan (1986, p. 10), Halle, Harris y Vergnaud (1991, p. 142), Devine y Stephens (1994, p. 267), Harris 1996 (pp.179-180).

3 En las glosas y en los diagramas, se emplean las siguientes abreviaciones: 1=primera persona, $2=$ segunda persona, $3=$ tercera persona, ac. $=$ acusativo, act. $=$ voz activa, cons. $=$ consonántico dat. $=$ dativo, gen. $=$ genitivo, imp. $=$ imperativo, impto. $=$ imperfecto, ind. $=$ indicativo, nom. $=$ nominativo, opt. $=$ optativo, pas. $=$ voz pasiva, pl. $=$ plural, post $=$ posterior, pte.=presente, red. $=$ redondeado, $R L A=$ raíz de la lengua avanzada, $s .=$ singular, subj. $=$ subjuntivo, voc. $=$ vocativo. Otras abreviaciones se explican en el texto.

4 Cf. Brandenstein (1964, pp. 90-91), De Haas (1988, pp. 97-99), Devine-Stephens (1994, pp. 17), Horrocks (2010, p. 161).

5 En el Cuadro 1 se emplean los signos de la ortografía convencional del griego. Todos los segmentos son vocales puras. Los valores de los mismos en el Alfabeto Fonético Internacional son: $\iota=[\mathrm{i}], \mathrm{v}=[\mathrm{y}], \varepsilon=[\mathrm{e}], \mathrm{o}=[\mathrm{o}], \alpha=[\mathrm{a}], \mathrm{\iota}(\mathrm{l} \#)=[\mathrm{i}:], v(\mathrm{\nu})=[\mathrm{y}:]$, $\varepsilon \mathrm{\varepsilon}=[\mathrm{e}:]$, ov $=[\mathrm{o}:], \eta=[\#:], \omega=[\#:], \alpha(\alpha \#)=[\mathrm{a}:]$.

6 Cf. Kühner-Blass (1890, pp. 212-218).

7 El acento es también analógico (cf. Smyth, 1920, p. 20, Grammont, 1948, p. 363, Brandenstein, 1964, p. 214).

8 Cf. Sommerstein (1973, pp. 56-59), De Haas (1988, p.140), Probert (2010, pp. 98-99).

9 Cf. De Haas (1988, p. 83), McCarthy-Prince (1996, pp. 41-43).

10 Cf. Waeschke (1875, p. 4), Grammont (1948, p. 354), Lejeune (1987, pp. 248-249), Devine-Stephens (1994, p. 267).

11 Cf. Waeschke (1875, p. 18), De Haas (1988, pp. 153-154).

12 Cf. Hayes (1989, p. 253), Halle (1995, p. 13), Harris (1996, p. 179), Rubach (1998, p. 177). Importa indicar que el valor cuantitativo o duración de la sílaba se mide en función de las ranuras asociadas con los núcleos.

13 Jakobson-Halle (1967, p. 30), De Haas (1988, p. 77), Clements (1990, sec. 6.4), Devine-Stephens (1994, p. 253), Casali (2011, p. 1449).

14 Cf. Grammont (1948, p. 359), Jakobson (1984, p. 53), Clements (1990, sec. 5.1), McCarthy-Prince (1996, p. 6).

15 Monro (1891, p. 83), Grammont (1948, p. 358), Palmer (1962, p. 95), Chantraine (1974, p. 756), Lejeune (1987, p. 244).

16 Cf. Brandenstein (1964, p. 91), Horrocks (2010, p. 161-162), Probert (2010, p. 97).

17 Cf. McCarthy (1986, p. 208), Yip (1988, p. 66), Halle (1995, p. 42).

18 Cf. La descripción de Grammont (1948, pp. 357-358) y la de Lejeune (1987, p. 248) con la de De Haas (1988), donde se afirma que las vocales largas medias cerradas (p. 127) y las vocales altas (p. 151), están excluidas del dominio de la contracción.

19 La representación plena de los segmentos es geométrica y jerárquica. En (21iii), (22), (23), y en general en el texto, los valores contrastivos del Cuadro 2 se dan en forma de lista. Pero propiamente están dominados por [-cons.] y se desprenden de otros nodos de la geometría de rasgos fonológicos (cf. Halle, 1995, p. 2).

20 Desde aquí, solo se representa en los diagramas las ranuras que cuentan para determinar la cantidad de la sílaba

21 Así, en (35), donde se suman cuatro tiempos el reajuste se hace por completo con la aplicación iterativa de Exceso de Moras.

22 Cf. Smyth (1920, 22), Grammont (1948, p. 379), De Haas (1988, p. 125), Devine-Stephens (1994, p. 267).

\section{BY-NC-SA}

